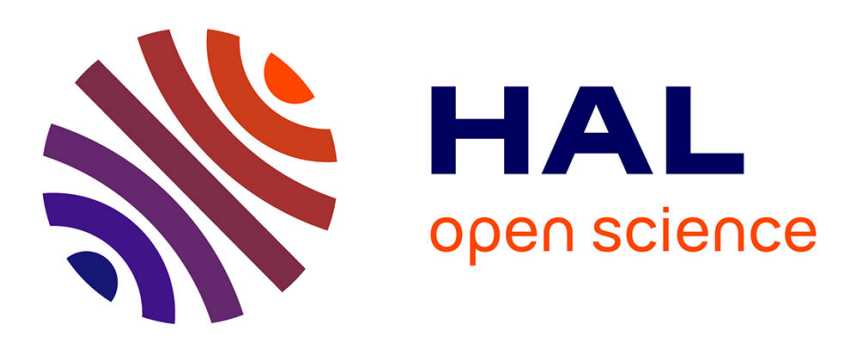

\title{
Interactions between excitation and extraction modes in an organic-based plasmon-emitting diode
}

Nan-Fu Chiu, Maxime Le Ster, Cheng-Du Yang, Ming-Hung Tseng, Feng-Yu Tsai

\section{- To cite this version:}

Nan-Fu Chiu, Maxime Le Ster, Cheng-Du Yang, Ming-Hung Tseng, Feng-Yu Tsai. Interactions between excitation and extraction modes in an organic-based plasmon-emitting diode. Applied Surface Science, 2015, 332, pp.97-104. 10.1016/j.apsusc.2015.01.148 . hal-01225593

HAL Id: hal-01225593

https://hal-univ-rennes1.archives-ouvertes.fr/hal-01225593

Submitted on 19 Nov 2015

HAL is a multi-disciplinary open access archive for the deposit and dissemination of scientific research documents, whether they are published or not. The documents may come from teaching and research institutions in France or abroad, or from public or private research centers.
L'archive ouverte pluridisciplinaire $\mathbf{H A L}$, est destinée au dépôt et à la diffusion de documents scientifiques de niveau recherche, publiés ou non, émanant des établissements d'enseignement et de recherche français ou étrangers, des laboratoires publics ou privés. 
Highlights :

- Directional emission properties give rise to a spectral band-gap response enhancement.

- The subsequent emission intensity can increase by up to 3.5 times.

- FWHM of approximately $60 \mathrm{~nm}$ in a defined direction is achieved.

- SP coupling rate is approximately $80 \%$ on the metal grating structure. 


\title{
Interactions between excitation and extraction modes in an organic based plasmon-emitting diode
}

\author{
Nan-Fu Chiu ${ }^{1, *}$, Maxime Le Ster ${ }^{1,2}$, Cheng-Du Yang ${ }^{1}$, Ming-Hung Tseng ${ }^{3}$, \\ Feng-Yu Tsai ${ }^{3}$ \\ ${ }^{1}$ Institute of Electro-Optical Science and Technology, National Taiwan Normal \\ University, Taipei 11677, Taiwan \\ ${ }^{2}$ Material Sciences and Engineering, Institut National des Sciences Appliquées \\ de Rennes, Rennes 35708, France \\ ${ }^{3}$ Department of Material Science and Engineering, National Taiwan University, \\ Taipei 10617, Taiwan
}

Corresponding author:

nfchiu@ntnu.edu.tw $\underline{T E L:} \underline{+886-2-77346722}$ FAX: $\underline{886-2-8663-1954}$ 


\section{Abstract:}

This study demonstrates the feasibility of enhancing an organic based plasmon-emitting diode on the directional light beaming efficiency by near-field surface plasmon polaritons (SPPs) in both metal grating and polymer grating nanostructures. The interaction between organic/metal and PR/metal interfaces to cause SPPs can facilitate specific directional emission. Directional emission properties give rise to a spectral band-gap response enhancement. Our results also verify that efficient surface plasmon grating coupled emissions (SPGCEs) can improve directionality under index mediated tuning. Experimental results indicate SP decoupling emission in the visible light. The subsequent emission intensity can increase by up to 3.5 times. Moreover, a narrow FWHM of approximately $60 \mathrm{~nm}$ in a defined direction is achieved, and a SP coupling rate is approximately $80 \%$ on the metal grating structure. The proposed is highly promising for use as an active plasmonic emitter and discoloration biosensors with enhanced SPPs resonance energy, owing to interactions with the organic/metal nanostructure.

Keywords: surface plasmon polaritons (SPPs), surface plasmon grating coupled emissions (SPGCEs), directional emission, full width at half maximum (FWHM), plasmonic emitter, biosensors. 


\section{Introduction}

Surface plasmon polaritons (SPPs) [1-4] are photons passing through a dielectric material and metal film surfaces causing their density to free electrons to resonate with energy transfer at the same wavevector as the coupling photon's wave. Most research in the recent decade has focused on increasing radiation efficiency [5-7], in which surface plasmon coupled emissions (SPCE) mechanisms from fluorescent molecules by incident wave excite an evanescent field near the Kretschmann configuration [8-10]. Recent efforts have implemented organic based plasmonic emitting diodes by using the surface plasmon grating coupled emissions (SPGCE) $[11,12]$ to develop related design concepts and technologies for sensing applications [13-17]. This SPGCE brings internal dipole interaction and light extraction mechanisms on the metal nanostructures $[18,19]$. While consisting of hybrid modes of light fields and oscillating electron fields at the metal/organic material interfaces, SPGCE uses the spontaneous emission of tris-(8hydroxyquinoline) aluminum $\left(\mathrm{Alq}_{3}\right)$ organic semiconductor molecules to excite surface plasmon resonance (SPR) by grating coupled emission. The emissions correspond to the resonant condition of surface plasmon (SP) modes on the organic/Au interface and grating coupled to the $\mathrm{Au} / \mathrm{air}$ interface for the emission of light. This light extraction passes through a metal approach to excite the evanescent wave on the periodic nanostructure, leading to a highly tunable emission property and increasing the luminescence efficiency.

This study develops different mechanisms based on SPR to compare the propagation property for the coupling effect of an electrical field and higher radiative emission rate. While our previous studies [11, 17] have addressed this question, certain aspects are explored in more detail in this study, especially surface plasmon band gap (SPBG) modes associated with both the excitation and extraction interaction [20,21]. An attempt is also made to more thoroughly elucidate the nature of all of the SPPs modes uncovered by 
examining the dispersion relation for a grating distribution at the resonance frequency for each mode. The proposed SPGCE method is highly promising for use in biosensor applications, owing to its high speed, label-free and high sensitivity capabilities with realtime reaction monitoring.

\section{Experimental methods and theoretical models}

This study has designed different plasmonic emitting devices by verifying a proposition involving the relationship between the SPR coupling effect of excitation and emission rate of extraction. In the device fabrication, the nanostructure with lamellar grating patterns is prepared by using electron-beam lithography (EBL). The grating pattern consists of 100 $\mathrm{nm}$ thick $\mathrm{Au}$ on $\mathrm{Si}_{-} \mathrm{Si}_{3} \mathrm{~N}_{4}$ substrate with an exposure area of $1.2 \times 1.2 \mathrm{~mm}^{2}$, as shown by four devices in Fig. 1. The pattern of grating pitch size of $500 \mathrm{~nm}$ consists of four devices of $\mathrm{Au}-$ film/Au(grating) for device-1 and Au-film/PR(grating) for device-2, and Aufilm/Au(grating)/Alq $\mathrm{q}_{3}$ for device-3 and $\mathrm{Au}$-film/PR(grating)/Alq $\mathrm{q}_{3}$ for device-4 structure, respectively. The device- 1 and device- 2 structures were without the organic $\mathrm{Alq}_{3}$ layer. The device-3 and device- 4 structures were fabricated by thermally evaporating organic $\mathrm{Alq}_{3}$ layer. The layer of $80 \mathrm{~nm}$ thick emissive $\mathrm{Alq}_{3}$ deposited by thermal evaporation under vacuum $\left(\sim 10^{-7}\right.$ Torr) to obtain the corrugated structures of the organic thin films. The details of the fabrication process were reported in our previous work [11, 17].

In this system setup, an angular interrogation-based SPR sensor system is designed for $\theta / 2 \theta$-mode (GWC Technologies, USA) and photoluminescence (PL) emission measurement system (Fig. 2) for angular fluorescence spectra-mode by using the designated SPGCE structure. These angle measurement systems are used to implement the SP absorption mode and SP emission mode spectra. This PL system has two kinds of light sources for a 405-nm laser diode light source (BWB-405-20E (20mW), B\&W TEK Inc, USA) and a white light source (Newport Oriel Spectral Luminator 69050) used to excite 
$\mathrm{Alq}_{3}$ molecules at a fixed incident angle of $45^{\circ}$ on the SPGCE device. A laser passes through a camera objective lens, iris diaphragms, polarizer filter and convex lenses, focuses on the device chip. Organic light emitters are pumped by laser directly, and we scan the photoluminescence from different angles. These devices are placed on a rotational stage, and a spectrum meter on the arm connected to the stage makes a revolution around the chip. The emissions of organic film on metal grating nanostructures are collected by a focal lens and analyzed by the high-resolution spectrometer (HR2000 UV-NIR, Ocean Optics Co.). The spectrometer is moved at an angle $\left(\theta_{e}\right)$ to determine the PL intensity of that specific emission angle in order to examine the variations of top-side emitted light.

\subsection{Theoretical models of grating coupled SPR (GCSPR)}

Briefly, the grating coupled SPR (GCSPR) [22, 23] is formed by a periodically symmetrical lamellar grating, (Figs. 1(a) and 1(b)). An incident single-wavelength of TM mode plane wave is separated, upon reflection, into different orders. When the grating period is shorter than the wavelength of light, the SP wave vector $\left(k_{S P}\right)$ is longer than the wave vector $(k / /)$ of the light and can comply with the SPR conditions in Eq. (1) and Eq. (2). These equations show the construction for the $k$ vectors of the plane waves. The angle of reflection of $m_{t h}$ order may be related to the angle of incidence, as obtained from Eq. (3). $[12,17,19]$

$$
\begin{aligned}
& k_{s p}= \pm k_{0} \sqrt{\frac{\varepsilon_{m} n_{e f f}^{2}}{\varepsilon_{m}+n_{e f f}^{2}}} \\
& k_{/ /}=n_{e f f} k_{0} \sin (\theta)=k_{S P} \pm m_{t h} \frac{2 \pi}{\Lambda} \\
& \sin \theta_{R}^{\left(m_{t h}\right)}=\sin \theta_{i}+\frac{m_{t h} \lambda}{\Lambda} \\
& n_{\text {eff }}=\sqrt{\varepsilon_{\text {eff }}}=\sqrt{\varepsilon_{\perp}}-\sqrt{\varepsilon_{\|}}
\end{aligned}
$$


where $\varepsilon_{m}$ is the permittivity of the thin gold film; $\theta_{i}$ is the angle of incidence; $\theta_{R}^{(m)}$ is the angle of reflection of $m_{t h}$ order and depends on wavelength; $\Lambda$ is the pitch of the grating; $n_{\text {eff }}[24]$ is the effective complex refractive index of the grating nanostructure; $\varepsilon \perp$ is the electrical field component orthogonal to the grating period dielectric constant; and $\varepsilon_{/ /}$is the electrical field component oriented parallel to the dielectric constant of the grating bars. Its behavior is observed through the excitation of surface plasmons, which are strong optical resonances based on coherent oscillations of the free electrons in the metal grating surface. Therefore, the reflected waves consist of an infinite sum of plane waves propagating at different angles with respect to the $\mathrm{z}$ axis. The SPR angles depend on the wavelength of the incident wave.

The GCSPR device is based on light diffraction on a periodically modulated surface of a nanostructure. Figure 3(a) shows the SPR dispersion relationship curve for a grating coupler $[22,25]$. A vector diagram of the various wave vectors is associated with the incident wavelength, the grating, the diffracted light, and the surface plasmon. When $\boldsymbol{\Lambda}$ to increase the $\theta^{(m t h)}=\theta_{i}$ or $\boldsymbol{\Lambda}$ to decrease the $\theta^{(m t h)}$ becomes imaginary, $k_{x}$ becomes a surface evanescent wave in $x$ axis propagation. An evanescent wave is an electromagnetic field with an imaginary momentum component in the direction $\mathrm{z}$ transverse to the direction of wave propagation $\mathrm{x}$, and with an imaginary wavenumber kz. Since the momentum is imaginary, a photon does not propagate towards z, despite the existence of the field. Under this circumstance, the surface plasmons are excited by -1 st order diffracted light. This coupling condition can be expressed as Eq. (5). Based on Eq. (5), the azimuthal angle at SP coupler occurs is given by Eq. (6). [19, 22]

$$
\begin{aligned}
& k_{S P}^{2}=n_{e f f}^{2} k_{0}^{2} \sin ^{2} \theta_{i}+\left(m_{t h} \frac{2 \pi}{\Lambda}\right)^{2} \pm 2 m_{t h} \frac{2 \pi}{\Lambda} k_{0} \sin \theta_{i} \cos \varphi \\
& \cos \varphi=\frac{\lambda}{2 n_{\text {eff }} \Lambda \sin \theta_{i}}\left|m_{1}+m_{2}\right|
\end{aligned}
$$


where $\varphi$ is an azimuthal angle, and $m_{1}$ and $m_{2}$ are the diffraction order to which the SP modes associated with them are coupled, where much of the evanescent light is confined.

\subsection{Theoretical models of SP grating coupled emission (SPGCE)}

The proposed SPGCE method is based on the fluorescent emission from the active organic layer and its diffraction in a periodically modulated surface with possible improvement of SPPs, owing to subwavelength grating of metallic thin films. Based on use of SPGCE device as a simplified model, Figs. 1(c) and 1(d) show the possible interaction mechanisms. The SPGCE method of a given surface plasmon photonic defines its optical properties, including transmission, reflection, and angular dependence. Spatial distribution of an electromagnetic field can be manipulated in a SP band-gap to improve the local field in a dielectric. Figure 3(b) shows the changes from non-radiative SPPs to radiative emission by the cross-coupling of grating nanostructure of specific direction optical properties under different diffraction orders and emission angles. According to this figure, the cross-coupled energy transfer is equivalent to near-field coupling of the dipole to SPPs modes in organic/metal interface. In the SPGCE theoretical model, at a certain wavelength, $(\lambda)$ can originate from the matching momentums of the internal emission $\left(\theta_{e}\right)$ through grating coupler condition (dielectric constant of $\mathrm{Alq}_{3}$ and pitch size of $\Lambda$ ) to the organic/metal $\left(k_{S P \text { (organic/metal) }}\right)$ dispersion curve, as shown in Eq. (7). Equation (8) is the wave-vectors parallel to the surface of the emission light. Moreover, Eq. (7) and Eq. (8) give such a matching condition for the guided mode of the organic layer and the coupling of light emission. SP-coupling emission occurs when the Bragg equation satisfies the SP wave resonance,

$$
k_{S P(\text { metal / organic })}=k_{0} \sqrt{\frac{\varepsilon_{\text {metal }} \cdot \varepsilon_{\text {organic }}}{\varepsilon_{\text {metal }}+\varepsilon_{\text {organic }}}}
$$


$\sin \left(\theta_{(\text {emission })}\right)=\frac{k_{S P}}{k_{0} \sqrt{\varepsilon_{\text {neff }}}} \pm m_{\text {th }} \frac{2 \pi}{k_{0} \Lambda}$

where $k_{s p}$ is the SP wave vector parallel to the surface of the interfaces between metal and dielectric (e.g., the organic/metal interface); $\varepsilon_{\text {metal }}$ is the permittivity of the thin gold film; and $\varepsilon_{\text {organic }}$ is the permittivity of the organic film. This property provides an effective approach for the dynamic control of light emission and absorption in SP modes. For the SPGCE theoretical model, a portion of light is propagated outwards through organic layers into the air. Therefore, Figures 1(a) and 1(b) show three wave vectors, i.e. $\mathrm{k}_{\text {radiative }}\left(k_{\text {rad }}\right)$,

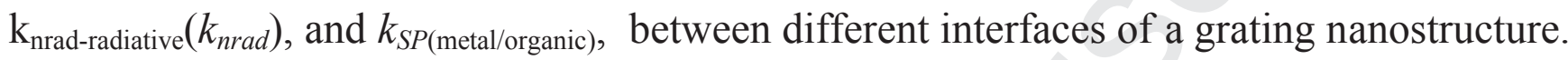
Such cross-coupling includes SPs and waveguide mode across the metal film.

\section{Results and discussion}

\subsection{Determination the resonance properties from the GCSPR structure}

This study implements the angular interrogation method in the $(\theta-2 \theta)$ mode of PL system for white light source. The angular modulation scheme adopts utilizes a zero order reflected configuration in which a range of angles on monochromatic light are incident onto the top side of the grating layer. The angle is scanned with a computer controlled motorized stage with a step size of 0.0025 . The GCSPR device is placed in the center of rotation of a high resolution rotary table measuring azimuthal angle $(\varphi)$. The out coupled beam is transmitted through a $2 \mathrm{X}$ telocentric lens, which has a working distance of $6.5 \mathrm{~cm}$ on a 1.2 Mega pixel Monochrome-CCD camera (The Imaging Source Europe GmbH). The camera determines coupled optical image change in ambient refraction index, leading to an observable shift of angle $(\theta)$ and intensity change in the reflectance.

Figure 4 shows the changes of angular-dependent reflectivity around resonant angle from the GCSPR device by using a TM polarized light source (PL system for white light source). The resonant peak for the grating coupler is measured on a rotary table at zero 
position (azimuthal angle $\varphi=0$ degree) with different incidence angles of reflected light $(\theta=$ 10 50 degrees). According to Figs. 4(a) for device-1 and 4(b) for device-2 structure, there is a minimum in the reflection of the difference wavelength light at a specific angle. The coupling efficiency is on the -1 st order of approximately $60 \%$ for device- 1 and $50 \%$ for device-2. Next, consider a situation, in the device-1 structure, when the incidence light of $630 \mathrm{~nm}$ produces the SPR angle on 10 degrees. Our results reveal a common phenomenon in which an increasing wavelength of incident light can produce a greater SP resonance angle. In the device-2 structure, the SP resonance characteristics of angle shift are opposite those of the device-1 structure. The SPR propagation loss occurs mainly owing to the surface roughness and the electric field characteristic of hetero-junction contact in the dielectric/metal interfaces [26].

In Figs. 4(c) for device-1 and 4(d) for device-2 structure show the analysis results of the SPR resonant angle $(\theta)$ with azimuthal angle $(\varphi)$. Comparing the measurement and calculation results reveals that the azimuthal angle characteristic exhibits SPR angle in angular interrogation for the azimuth values $\varphi$ from 0 to 50 degrees. This study also confirms the existence of SPP by calculating the measurement results of the theoretical model of grating coupler on a thin layer of metal with the momentum-matching condition of a GCSPR in our experimental configuration.

\subsection{Determination of the resonance properties from the SPGCE structure}

Photoluminescence (PL) measurement for white light source is taken for the specific directional resonance spectra produced by a designated SPGCE device. This experiment was performed at Glovebox Systems for operationally high purity levels $<1$ PPM of $\mathrm{O}_{2}$ and $\mathrm{H}_{2} \mathrm{O}$ at room temperature. The SPGCE device can be significantly enhanced from the three mechanisms, i.e., coupled SPP from $\mathrm{Alq}_{3}$ scattering emission, non-radiative mode, and excitation of the waveguide mode in internal metal nanostructures. This study also 
discusses these mechanisms of such enhancement and tunability from organic molecules on a grating nanostructure via the SPGCE device.

In SPGCE devices, these structures of lamellar grating patterns use the electron-beam lithography fabrication procedure. Next, thin films of $\mathrm{Alq}_{3}$ for $80 \mathrm{~nm}$ were fabricated via thermal evaporation. The side-view of Figs. 5(a) and 5(b) shows the scanning electron micrograph (SEM) of the SPGCE devices. The cross sections are the device-3 and device-4 structures.

The excitation of SPGCE resonance spectra is monitored using the above angular interrogation measurement mode for PL system.

This PL system is a white light source used to excite $\mathrm{Alq}_{3}$ molecules at different incident angles on the SPGCE device. Figure 6 shows the different incident angles at a specific resonance absorption capacity. $500 \mathrm{~nm}$ of the pitch size allows for the otherwise absorption SPR mode to be coupled by the SPGCE diffraction condition. The spectral position of this resonance is governed by the pitch size and shape and by the dielectric functions of both the metal and the surrounding media [17, 26, 27]. Organic layer $\mathrm{Alq}_{3}$ and periodical structures are used as the medium to demonstrate control over SPPs propagation on the SPGCE structures. Figures 6(a) and 6(c) show different incident angles from 10 to 30 degrees to analyze the wavelength resonant spectral characteristics. Figures 6(b) and 6(d) show a 3D dispersion relation and resonance absorption characteristics. Its behavior includes dipole-dipole coupling between the metal and organic interference of the plasmons energy transfer. In Figs. 6(a) and 6(b), the device-3 nanostructure shows a wavevector of $-1^{\text {st }}$ order for $\mathrm{SPP} 1\left(\mathrm{Au} / \mathrm{Alq}_{3}\right)$ interfaces, which closely corresponds to the SPR energy modes. In Figs. 6(c) and 6(d), although having resonant spectral response characteristics, the device-4 nanostructure shows three different orders at $-1^{\text {st }}$ order for SPP1 $\left(\mathrm{Au} / \mathrm{Alq}_{3}\right), \quad \mathrm{SPP} 2(\mathrm{Au} / \mathrm{PR})$, and $-2^{\text {nd }}$ order for $\mathrm{SPP} 3\left(\mathrm{Au} / \mathrm{Alq}_{3}\right)$ interfaces SPR 
absorption. A defect in the grating structure results in strong absorption in visible light range and produces error wave propagation. Figures $6(\mathrm{c})$ and $6(\mathrm{~d})$ show the error wave paths, which are P1a, P1b, P2a and P2b points.

Figures 7(a) and 7(b) show the different incident wavelengths (using the PL system for white light source) from 480 to $660 \mathrm{~nm}$ and analysis of the resonance angle characteristics. Figure 7(a) shows the different wavelength light irradiation in the device-3 nanostructure from 480 to $560 \mathrm{~nm}$, in which this wave propagation ranges from 35 to 10 degrees. Obviously, the experimental results correlate well with Fig. 6 (b) white line (m) of $-2^{\text {nd }}$ order for SPP2(Au/Alq $)$ mode. Figure 7(b) indicates that the in device-4 nanostructure has two absorption peaks in the small angle of 10 15 degrees and a large angle of 25 35 degrees, respectively. Figure 6(d) shows these two absorption peaks for the propagation path that closely corresponds to the first-order curve of white line (n) of $-1^{\text {st }}$ order for $\mathrm{SPP} 1\left(\mathrm{Au} / \mathrm{Alq}_{3}\right)$ and $(\mathrm{O})$ of $-1^{\text {st }}$ order for SPP2(Au/PR) modes.

\subsection{Determination of the emission properties from the SPGCE structure}

Using the PL method of 405-nm laser that excites SPPs enhances the radiative light and control of the directional emission. Light emission scattering is used to excite an organic layer by surface plasmon on a thin gold film support. The dispersion relation for the scattering light arises from the momentum match between the $\mathrm{Au} / \mathrm{Alq} \mathrm{q}_{3}$ and $\mathrm{Au} / \mathrm{air}$ regions of the SPGCE device.

Figure 8 shows the behaviors of the SPP at a metal/organic interface via coupler transfer emission for the device-3, device-4 and planar structure. A planar structure has a maximum at $530 \mathrm{~nm}$ and a full width at half maximum (FWHM) of $120 \mathrm{~nm}$, as is expected for $\mathrm{Alq}_{3}$ emission. The SPGCE device results in a shape angular pattern of the emission, 
subsequently beaming most of the light into a very narrow angular range of approximately $60 \mathrm{~nm}$ of FWHM in a defined direction. The emission angle depends on the index of refraction at the organic/metal or metal/dielectric-layer interfaces. The SP coupler emission intensity can increase by up to 3.5 times. The SPP coupling effect increases the PL peak intensity along with an increase in which the times ratio is 1:2.5:3.5 for planar, device-3 and device-4. The device-3 structure contains two resonant emission peaks, representing the $-1^{\text {st }}$ order mode of the metallic slab. The first resonance angle for $-20,-15$ and -10 degrees at $\lambda_{\mathrm{em}}=520.43 \mathrm{~nm}, 540 \mathrm{~nm}$ and, $526.7 \mathrm{~nm}$, while the second resonance angle for -20 , -15 and -10 degrees at $\lambda_{\mathrm{em}}=645.7 \mathrm{~nm}, 672.3 \mathrm{~nm}$ and $698.6 \mathrm{~nm}$, excites the $-1^{\text {st }}$ order mode. Experimental results indicate that these devices have a strong directional emission as well as enhanced intensity. A preliminary estimate is made of the pitch or wavelength of the grating required to scatter the surface plasmon mode back out to light. Above results can be calculated from the SP dispersion relation in Eqs. (7) and (8). This high directional is explained as a combination of an increased efficiency in the excitation of the organic layer and an enhanced spatial coherence, which is achieved by coupling this organic layer to extended plasmon modes in the SPGCE nanostructure, and the subsequent out-coupling to free-space radiation.

Figure 9 shows the SPGCE device resultant of improved luminescence from multiple emission angles vs. SP resonance wavelength spectrogram. Figure 9(a) clearly shows the $\mathrm{SPP}_{1}\left(\mathrm{Au} / \mathrm{Alq}_{3}\right)$ coupled via a Bragg scatter of $-\Delta \mathrm{k}(-2 \pi / \Lambda)$ for $-1^{\text {st }}$ order dispersion relation. To ensure that the emission is a continuous function of wavelength, this phenomenon exhibits an over coupling (without gap). Figure 9(b) shows the emission dispersion relation $(\omega-\mathrm{k})$ at different grating pitch sizes of SPGCE devices by extracting the maximum intensities of PL spectra at different viewing angles. 
Figure 9(c) shows the device-4 nanostructure emission properties with a gap of $30 \mathrm{~nm}$ (SPBG-1). Its gap is via a Bragg scatter for -1st order to provide an SPP1(Au/Alq3) interaction between the branches scattered. The other a $-\Delta \mathrm{k}$ component for $-1^{\text {st }}$ order to provides the SPP2(Au/PR) branches scattered on SPBG-2 of $12 \mathrm{~nm}$. Figure 9(d) summarizes the experimental results of dispersion relation curves for the device- 4 structure at different viewing angles. The branches scattered for forward and backward traveling SP modes represent energy gap, which opens up for light at normal emission. The emission optical far-field patterns exhibit the symmetry and shape of the SP mode. The phenomenon is attributed to a metal surface plasmon mediated light out-coupling mechanism, which is also responsible for the relatively high extraction efficiency [20,21, 28].

Above measurement results demonstrate that the absorption (Figs. 6 and 7) and emission (Figs. 8 and 9) can be more significant than that of SP coupling in terms of the contribution of SP coupling if the $\mathrm{Alq}_{3}$ dipole emission $(480 \sim 680 \mathrm{~nm})$ can match the SP propagating behavior. This energy effect is attributed to SP excitation modes with respect to extraction associated with a different dispersion relation distribution. However, the SPPs tuning energy can be extracted as light by providing a grating nanostructure. Moreover, SPGCE may be SP coupler mechanisms with such emitter characteristics.

Figures 8 and 9 of the SPPs absorption demonstrate that this absorption exhibits coupler emission in the visible light range. However, also in this case, a surface plasmon mediated extraction mechanism plays an important role in the mode distribution and the overall decoupled efficiency of the emitter. The interaction between surface plasmons and tightly bound organic excited states, or excitons, must be thoroughly understood to determine the promising SPGCE device architecture for plasmonic organic emitter. 


\section{Conclusion}

SPGCE still plays an important role in studying the unique nanostructure characteristics of SPPs resonance modes with the interactions between electron fields on both sides of the metal interfaces. This study has demonstrated of the effectiveness of the metal and PR grating structure of the SP coupling rate and the azimuthal angle resonance characteristics. This study thoroughly investigates the lamellar gratings in the visible spectrum emission. Experimental results indicate that device- 3 and device- 4 contribute differently in terms of SP band gap with directional emission as well as enhanced intensity. The resulting emission intensity can reach up to 3.5 times, and has a very narrow angular range of about $60 \mathrm{~nm}$ of FWHM. In summary, the above results are highly promising for use as highly directional emitter and fluorescence biosensor with enhanced resonance energy, owing to interactions on the organic/metal grating nanostructure.

\section{Acknowledgements}

The authors would like to thank the Ministry of Science and Technology of the Republic of China, Taiwan, for financially supporting this research under Contract No. MOST 1032221-E-003 -008, NSC 102-2221-E-003-021, and NSC 99-2218-E-003-002-MY3. 


\section{References}

1. R. W. Wood, On a remarkable case of uneven distribution of light in a diffraction grating spectrum, Phil. Mag. 4 (1902) 396-408.

2. A. Otto, Excitation of nonradiative surface plasma waves in silver by the method of frustrated total reflection, Z. Phys. 216 (1968) 398-410.

3. E. Kretschmann, The Determination of the Optical Constants of Metals by Excitation of Surface Plasmons, Z. Phys. 241 (1971) 313-324.

4. J. Homola, I. Koudela and S. S. Yee, Surface plasmon resonance sensors based on diffraction gratings and prism couplers: sensitivity comparison, Sens. Actuators B Chem. 54 (1999) 16-24.

5. D. Gifford, D. G. Hall, Extraordinary transmission of organic photoluminescence through an otherwise opaque metal layer via surface plasmon cross coupling, Appl. Phys. Lett. 80 (2002) 3679-3681.

6. S. Wedge, W. L. Barnes, Surface plasmon-polariton mediated light emission through thin metal films, Opt. Exp. 12 (2004) 3673-3685.

7. J. Feng, T. Okamoto, S. Kawata, Highly directional emission via coupled surfaceplasmon tunneling from electroluminescence in organic light-emitting devices, Appl. Phys. Lett. 87 (2005) 241109.

8. J. R. Lakowicz, J. Malicka, I. Gryczynski, and Z. Gryczynski, Directional surface Plasmon-coupled emission: a new method for high sensitivity detection, Biochem. Biophys. Res. Commun. 307 (2003) 435-439.

9. G. Winter and W. L. Barnes, Emission of light through thin silver films via near-field coupling to surface plasmon polaritons, Appl. Phys. Lett. 88 (2006) 051109.

10.S.-H. Cao, W.-P. Cai, Q. Liu, and Y.-Q. Li, Surface Plasmon-Coupled Emission: What Can Directional Fluorescence Bring to the Analytical Sciences?, Ann. review of Anal. Chem., 5 (2012) 317-336. 
11.N.-F. Chiu, C. Yu, S.-Y. Nien, J.-H. Lee, C.-H. Kuan, K.-C. Wu, C.-K. Lee, and C.W. Lin, Enhancement and tunability of active plasmonic by multilayer grating coupled emission, Opt. Exp. 15 (2007) 11608-11615.

12.S. Y. Nien, N. F. Chiu, Y. H. Ho, J. H. Lee, C. W. Lin, K. C. Wu, C. K. Lee, J. R. Lin, M. K. Wei, and T. L. Chiu, Directional photoluminescence enhancement of organic emitters via surface plasmon coupling, Appl. Phys. Lett. 94 (2009) 103304.

13.Y. Wang, W. Knoll, J. Dostalek, Bacterial pathogen surface plasmon resonance biosensor advanced by long range surface plasmons and magnetic nanoparticle assays, Anal Chem. 84 (2012) 8345-50

14.W.-H. Yeh and A. C. Hillier, Use of dispersion imaging for grating-coupled surface plasmon resonance sensing of multilayer langmuir-blodgett films, Anal. Chem. 85 (2013) 4080-4086.

15.J.S. Yuk, E.F. Guignon and M.A. Lynes, Highly sensitive grating coupler-based surface plasmon-coupled emission (SPCE) biosensor for immunoassay, Analyst. 138(2013) 2576-2582.

16.N.-F. Chiu, T.-Y. Huang, C.-C. Kuo, C.-W. Lin, J.-H. Lee, Organic-based plasmonic emitters for sensing applications, Appl Opt. 52 (2013) 1383-1388.

17.N.-F. Chiu, C.-J. Cheng and T.-Y. Huang, Organic Plasmon-Emitting Diodes for Detecting Refractive Index Variation, Sensors, 13 (2013), 8340-8351.

18.D.M. Koller, A. Hohenau, H. Ditlbacher, N. Galler, F. Reil, F.R. Aussenegg, A. Leitner, E.J.W. List and J.R. Krenn, Organic plasmon-emitting diode, Nature Photonics 2 (2008) $684-687$.

19.N.-F. Chiu, C.-W. Lin, J.-H. Lee, C.-H. Kuan, K.-C. Wu, and C.-K. Lee, Enhanced luminescence of organic/metal nanostructure for grating coupler active long-range surface plasmonic device, Appl. Phys. Lett. 91 (2007) 083114. 
20.A. J. Benahmed and C.-M. Ho, Bandgap-assisted surface-plasmon sensing, Appl Opt. 46 (2007) 3369-3375.

21.M. Toma, K. Toma, P. Adam, J. Homola, W.Knoll, and J. Dostálek, Surface plasmoncoupled emission on plasmonic Bragg gratings, Opt. Exp. 20 (2012) 14042-14053.

22.F. Romanato, K. H. Lee, H. K. Kang, G. Ruffato, and C. C. Wong, Sensitivity enhancement in grating coupled surface plasmon resonance by azimuthal control, Opt. Exp. 17 (2009) 12145-12154.

23.K. Tawa, H. Hori, K. Kintaka, K. Kiyosue, Y. Tatsu, and J. Nishii, Optical microscopic observation of fluorescence enhanced by grating-coupled surface plasmon resonance, Opt. Exp. 16 (2008) 9781-9790.

24.S. Sinzinger and J. Jahns, Microoptics $2^{\text {nd }}$ ed., Wiley-Vch, Weinheim, 2003.

25.A. Giannattasio and W.L. Barnes, Direct observation of surface plasmon-polariton dispersion, Opt. Exp. 13 (2005) 428-434.

26.C.-W. Cheng, M. N. Abbas, M.-H. Shih, Y.-C. Chang, Characterization of the surface plasmon polariton band gap in an $\mathrm{Ag} / \mathrm{SiO} 2 / \mathrm{Ag} \mathrm{T}$-shaped periodical structure, Opt. Exp. 19 (2011) 23698-23705.

27.P. Andrew, W. L. Barnes, Energy Transfer Across a Metal Film Mediated by Surface Plasmon Polaritons, Science 306 (2004) 1002-1005.

28.S. Wedge, A. Giannattasio, W.L. Barnes, Surface plasmon-polariton mediated emission of light from top-emitting organic light-emitting diode type structures, Organic Electronics 8 (2007) 136-147. 


\section{Figure Captions:}

Figure 1. Schematic diagrams of the working mechanism of the grating structures. The effect of non-radiative modes by Bragg scatting could be utilized the high-efficiency coupling of far-field optical emission at some directional angles. The SPP's E-fields originate on from positive charges and terminate on negative ones. The samples are a grating coupled SPR (GCSPR) device for the (a) $\mathrm{Si}_{-} \mathrm{Si}_{3} \mathrm{~N}_{4}$ substrate /Au-film $(100 \mathrm{~nm}) / \mathrm{Au}-$ grating $(50 \mathrm{~nm})$ for device-1 structure and (b) $\mathrm{Si}_{-} \mathrm{Si}_{3} \mathrm{~N}_{4}$ substrate $/ \mathrm{Au}$-film $(100 \mathrm{~nm}) / \mathrm{PR}-$ grating $(100 \mathrm{~nm})$ for device-2 structure. The samples are an SP grating coupled emission (SPGCE) device for the (c) Si-Si3N4 substrate /Au-film $(100 \mathrm{~nm}) / \mathrm{Au}$-grating $(50 \mathrm{~nm}) / \mathrm{Alq}_{3}$ $(80 \mathrm{~nm})$ for device-3 structure. (d) $\mathrm{Si}_{-} \mathrm{Si}_{3} \mathrm{~N}_{4}$ substrate $/ \mathrm{Au}$-film $(100 \mathrm{~nm}) / \mathrm{PR}$-grating (100 $\mathrm{nm}) / \mathrm{Alq}_{3}(80 \mathrm{~nm})$ for device-4 structure.

Figure 2. Photograph of photoluminescence (PL) emission measurement system setup (1. diode laser of wavelength $405 \mathrm{~nm}, 2$. reflective mirror, 3. objective lens, 4. iris diaphragms, 5. polarizer filter, 6 convex lenses, 7. rotational stage, 8. device chip and sample holder, 9. focal lens, and 10. spectrum meter).

Figure 3. Surface plasmon polariton dispersion curves. (a) coupling phase definition of the GCSPR model propagating at a free metal-air interface. (b) Dispersion curve of the SPGCE model for emission phase definition, the explained that intrinsic $\mathrm{Alq}_{3}$ emission and excitation into the $\mathrm{Au} / \mathrm{Alq}_{3}$ coupler SP emission angle.

Figure 4. Measurement of the reflectance intensity vs resonance angle from the device-1 structure for (a) and device-2 structure for (b) at TM-polarized light. SP resonance angle shift at a difference azimuthal angle with incident light wavelength (c) device-1 (d) device2 structures. 
Figure 5. SEM images of the SPGCE structure cross section, which display the arrangement of a periodically lamellar layer (a) device-3 structure of $\mathrm{Si}_{-} \mathrm{Si}_{3} \mathrm{~N}_{4}$ substrate /Au-film (100 nm)/Au-grating $(50 \mathrm{~nm}) / \mathrm{Alq}_{3}(80 \mathrm{~nm})$ and (b) device-4 structure of $\mathrm{Si}_{-} \mathrm{Si}_{3} \mathrm{~N}_{4}$ substrate /Au-film $(100 \mathrm{~nm}) /$ PR-grating $(100 \mathrm{~nm}) / \mathrm{Alq}_{3}(80 \mathrm{~nm})$ on a $500 \mathrm{~nm}$ pitch size.

Figure 6. SPGCE results of using SP-coupling to associate them with dispersion relation characteristics. Figures 6(a) and 6(c) show the SPGCE coupler on difference incident light wavelength of the resonance angle for device-3 and device-4 nanostructure, respectively. The spectra of SP resonance at angle of incident $\theta=10^{\circ}, 15^{\circ}, 20^{\circ}, 25^{\circ}$ and $30^{\circ}$, respectively. Figures 6(b) and 6(d) show 3D spectra for the incident at different wavelengths to analyze the resonance angle characteristics of device- 3 and device- 4 nanostructure, respectively.

Figure 7. (a) device-3 structure show $-1^{\text {st }}$ order field of SP energy with strong absorption curves, (b) device-4 structure show -1 st and -2 nd orders at $\mathrm{Au} / \mathrm{Alq}_{3}$ and $\mathrm{Au} / \mathrm{PR}$ interfaces of SP energy absorption curves.

Figure 8. PL emissions obtained from a SPGCE and planar structure use a 405-nm laser to excite an organic layer. The PL is measured on organic/metal grating. The changes in SPR are measured from the color of different angular spectra.

Figure 9. Experimental measurement of the emission spectra on observation angle $(\theta)$ as shown in (a) for device-3 and (b) for device-4 structure shows 3D contour plot of PL spectra (from 430 to $800 \mathrm{~nm}$ ) at different viewing angles (from $-40^{\circ}$ to $40^{\circ}$ ). (c) for device3 and (d) for device-4 structure as shown in dispersion relation $(\omega-k)$ with different pitch size. 

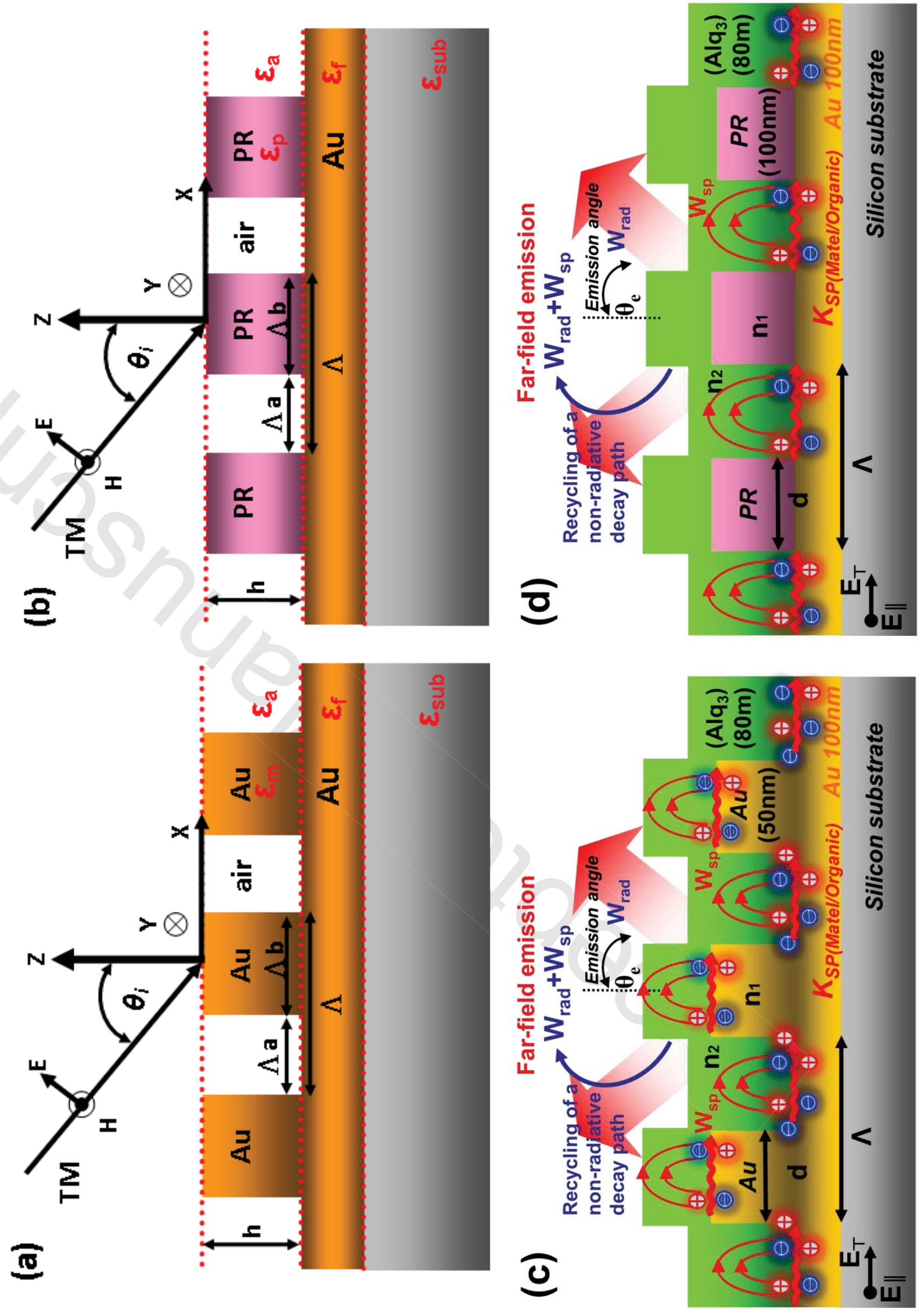

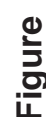




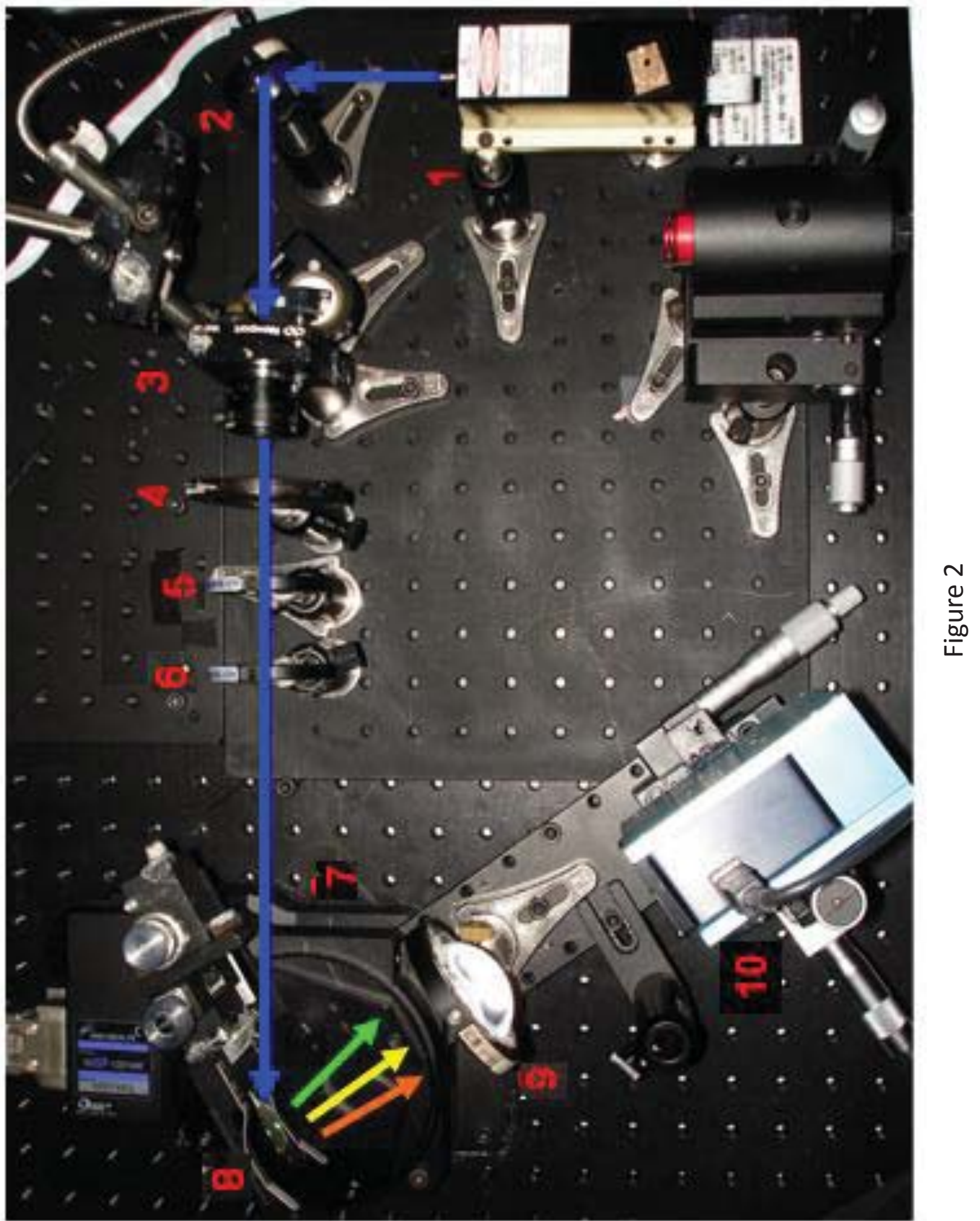



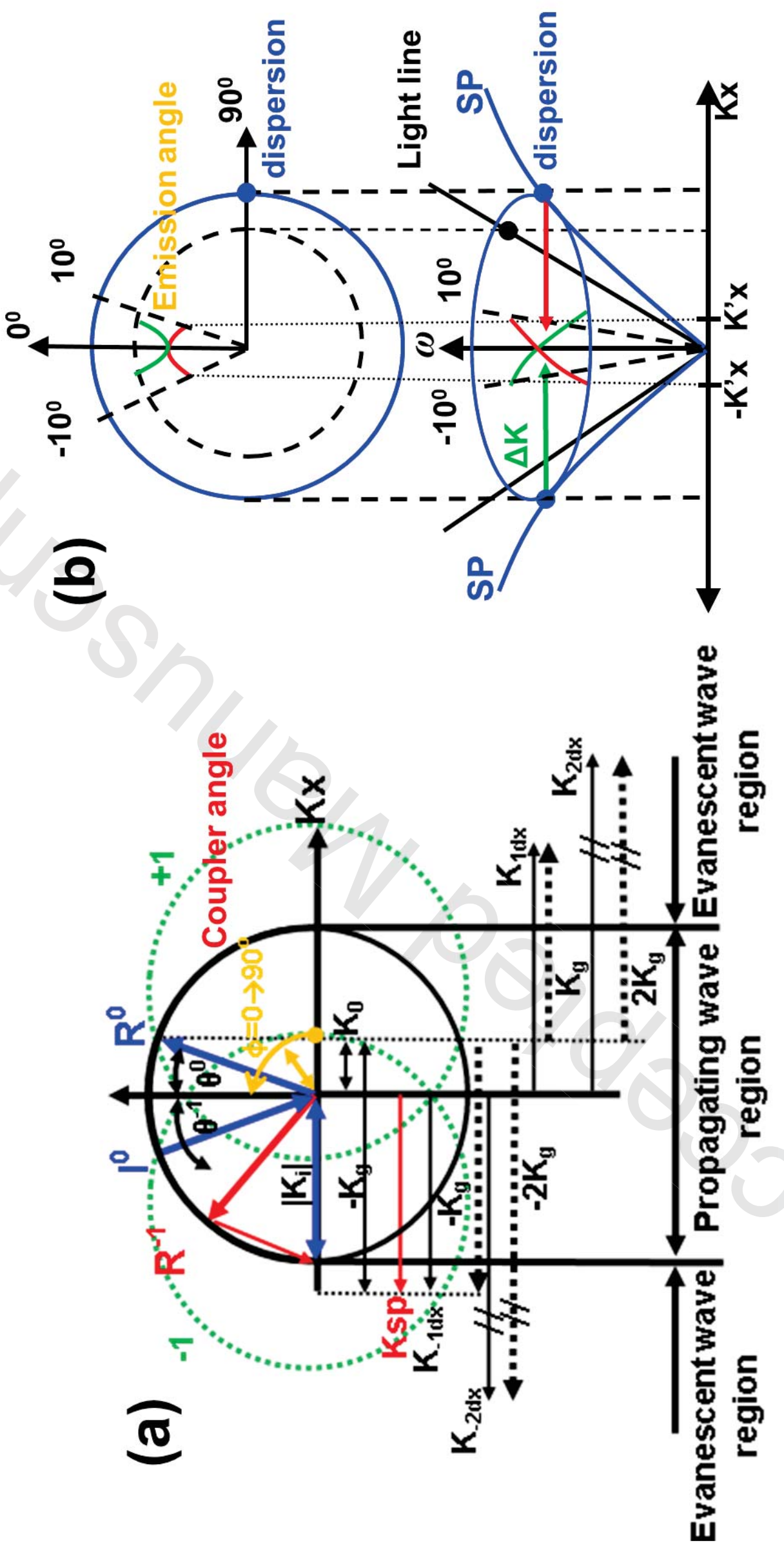


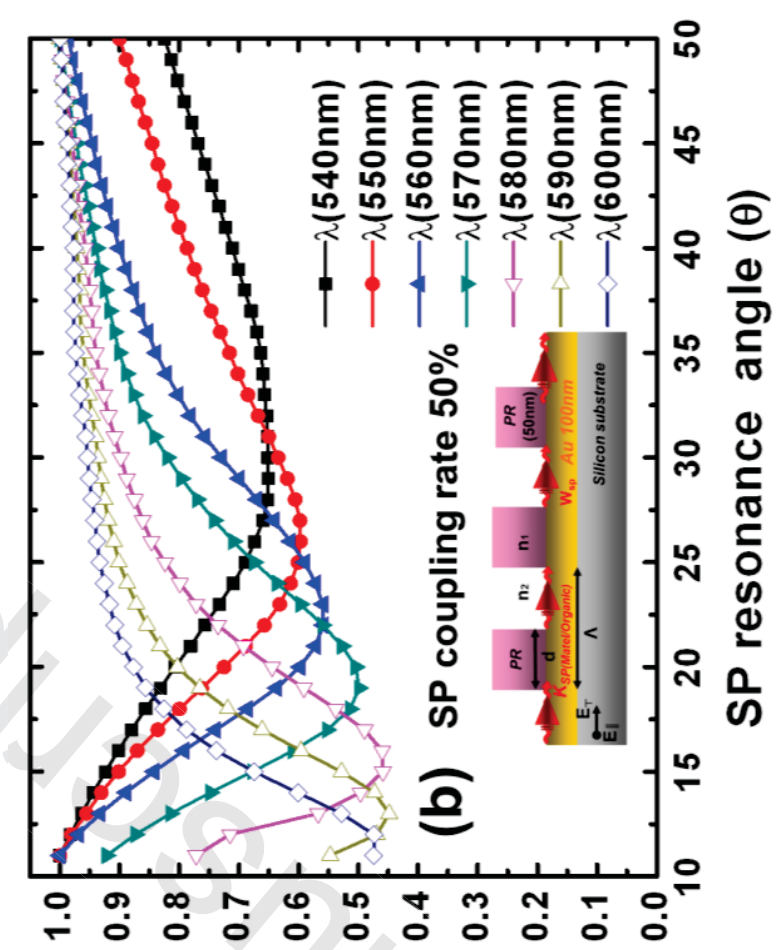

('n'e) К!

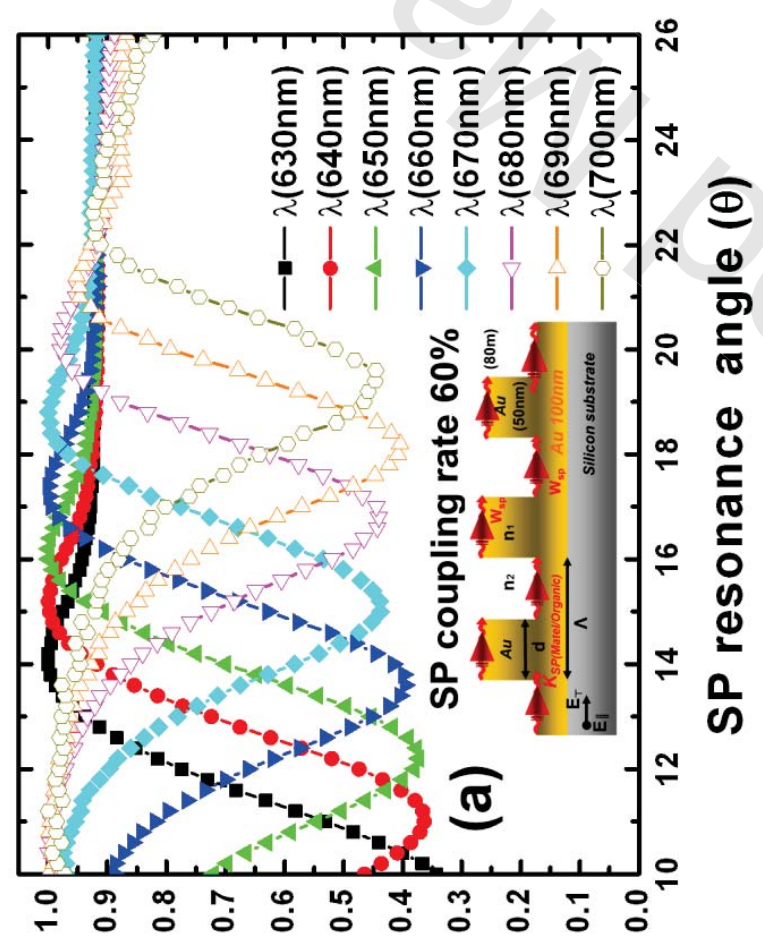

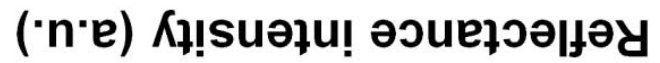

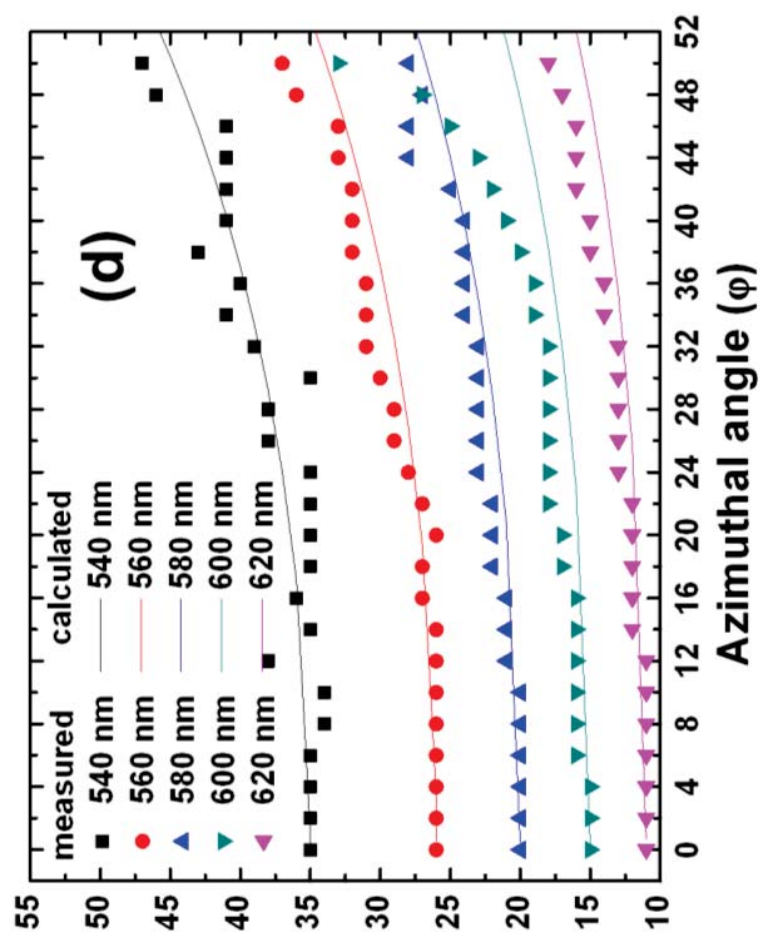

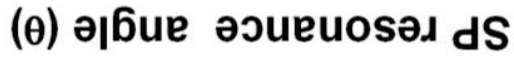

ป 

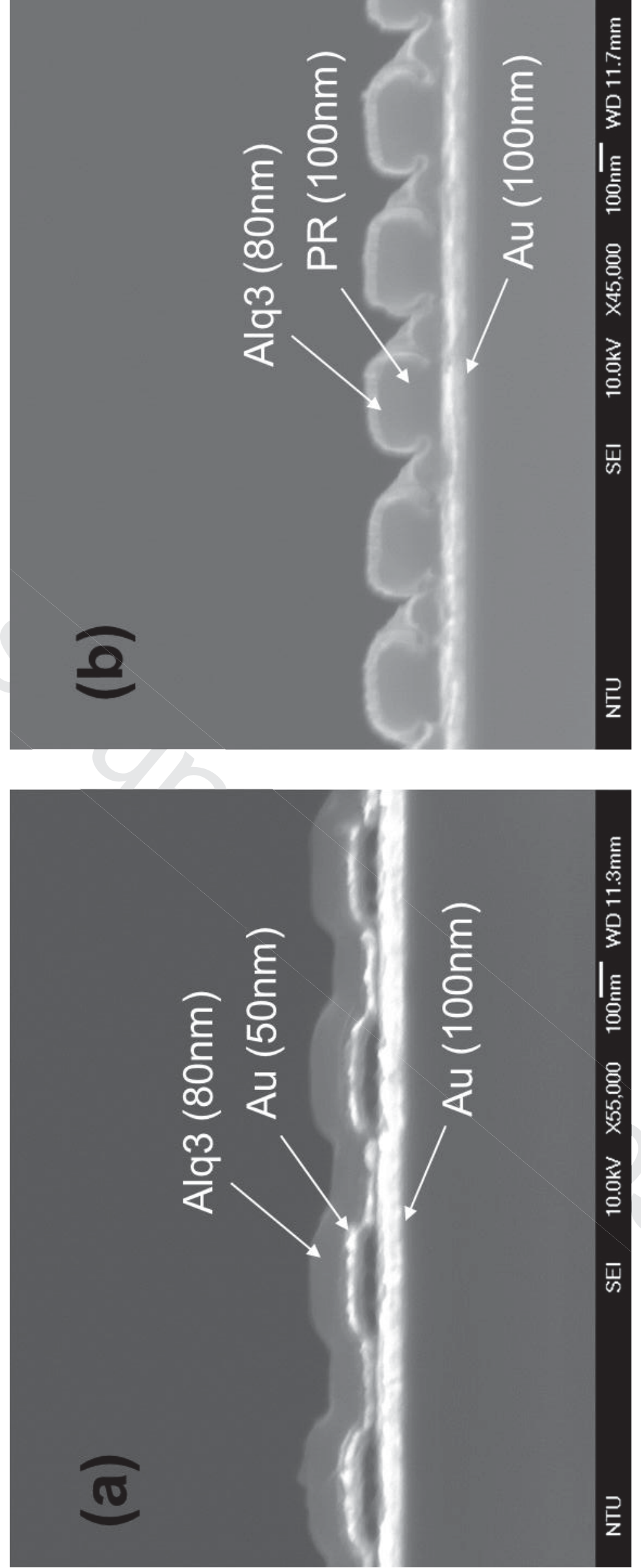

는 


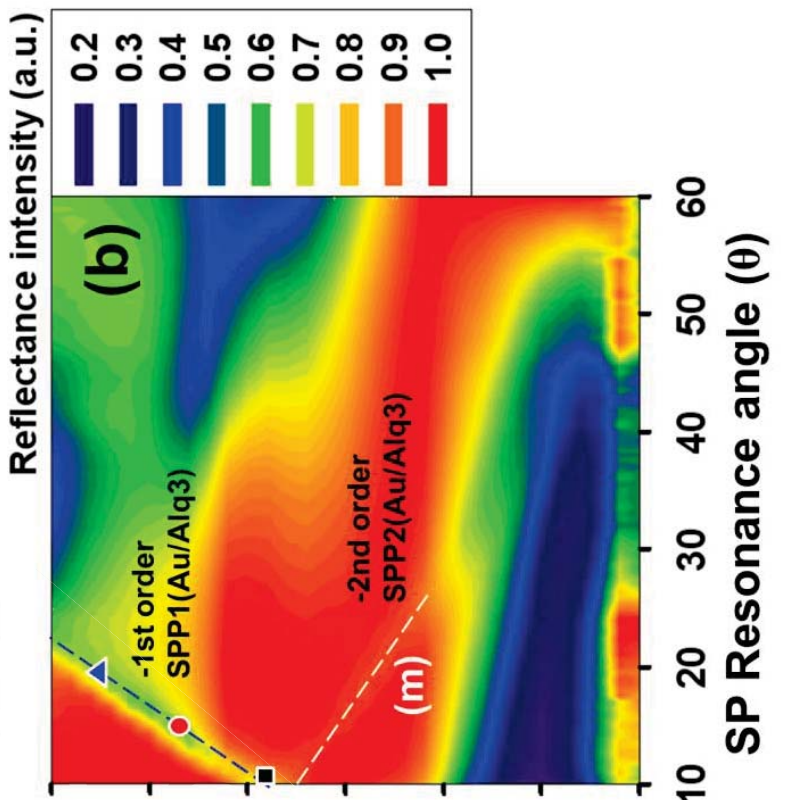

용 웅 (uu) чұбиәрәлем әэиеиоsәу dS

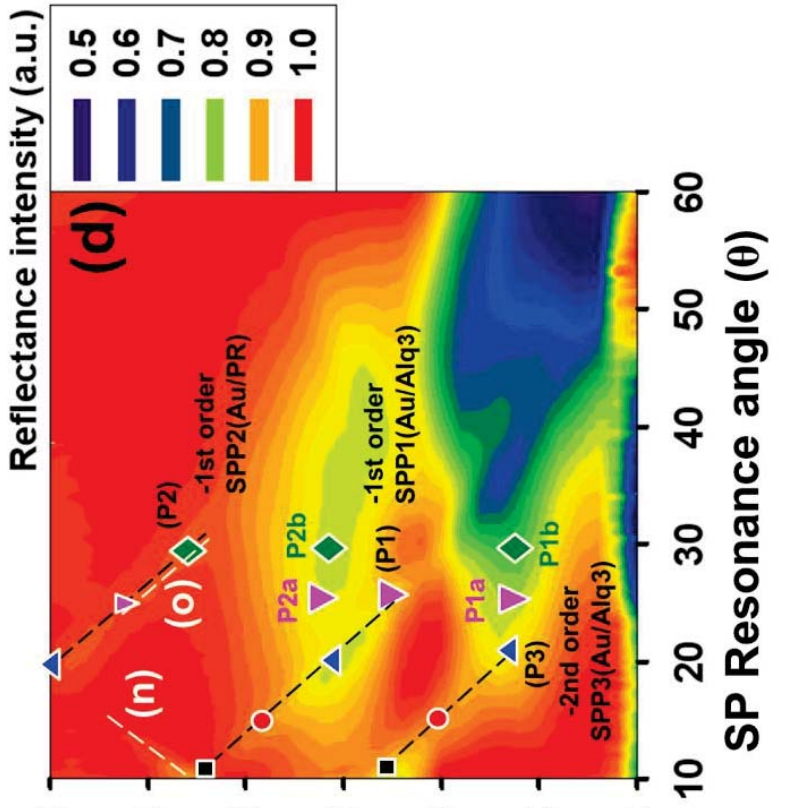

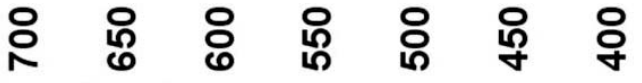

(uи) чұбиәрәкем әэиеиоsәу dS
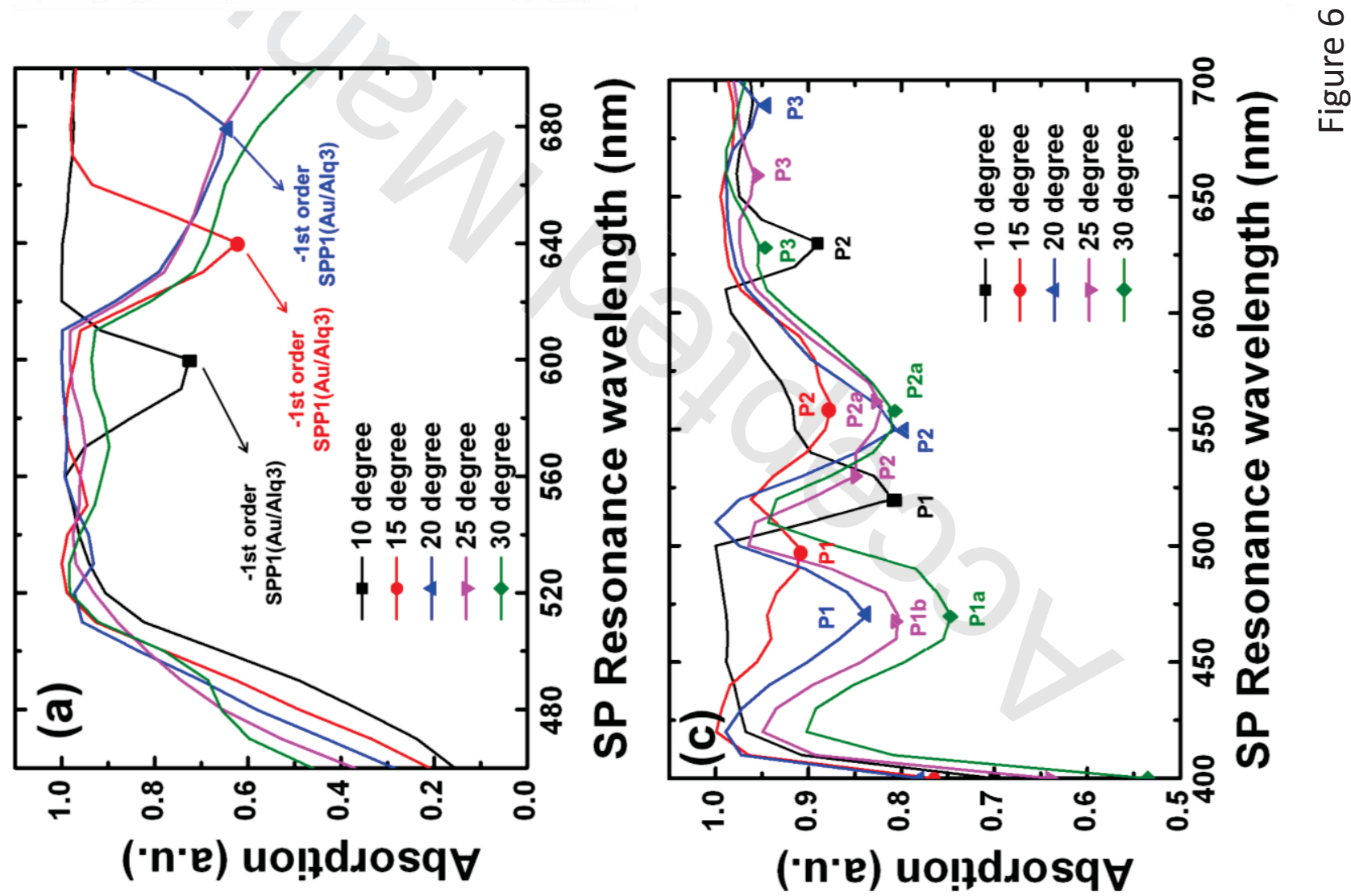
\begin{tabular}{l} 
N \\
4 \\
0 \\
$N$ \\
0 \\
\multirow{0}{0}{} \\
0
\end{tabular}
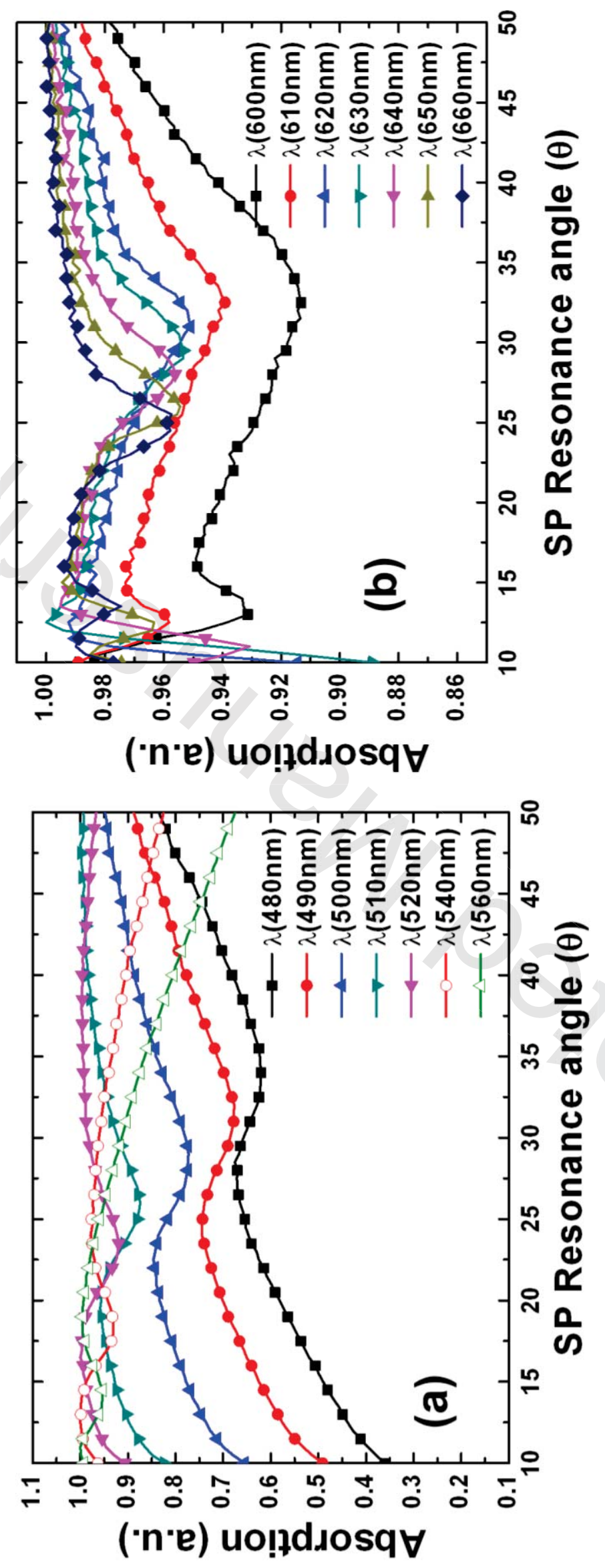

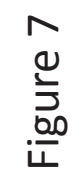




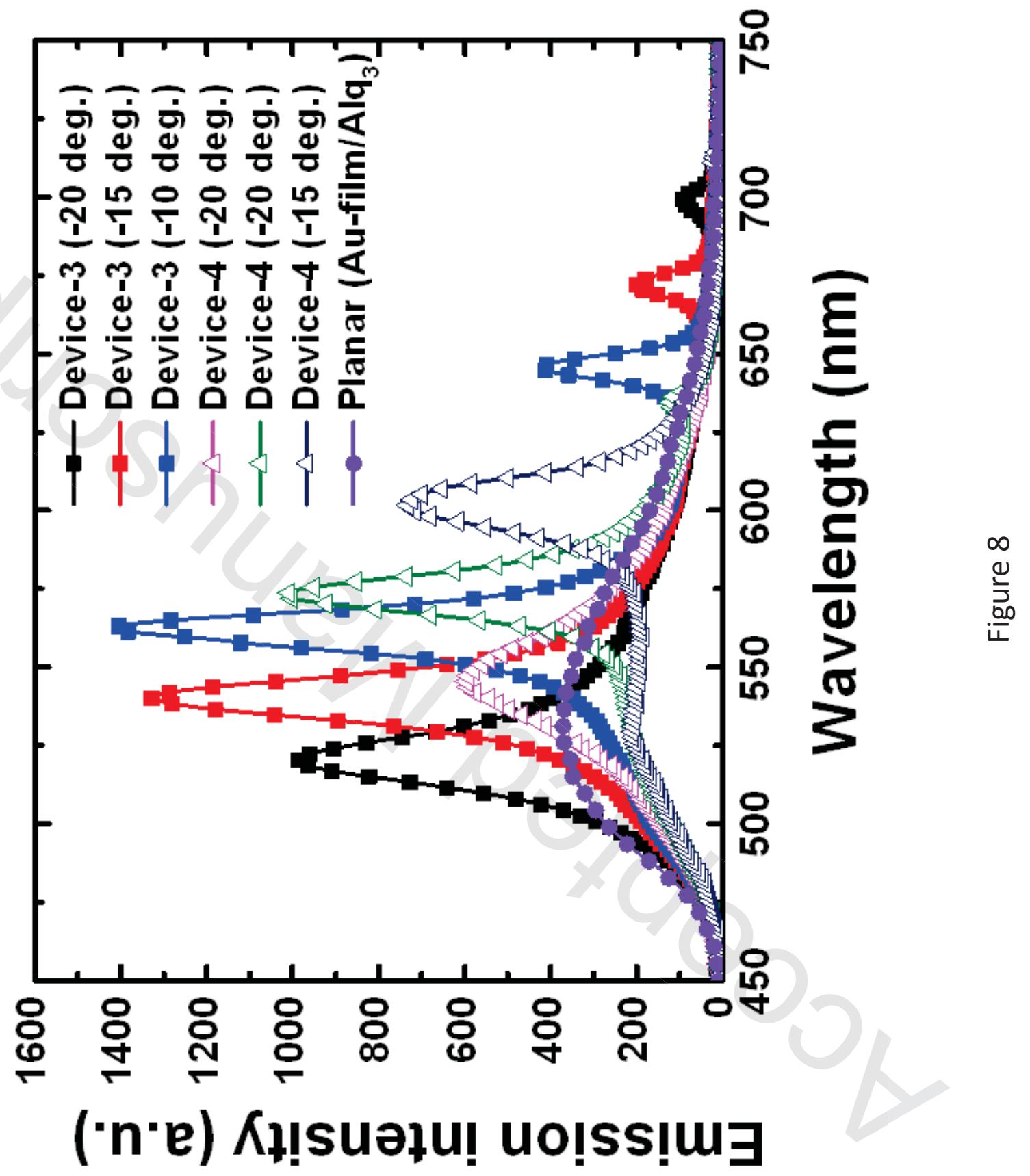



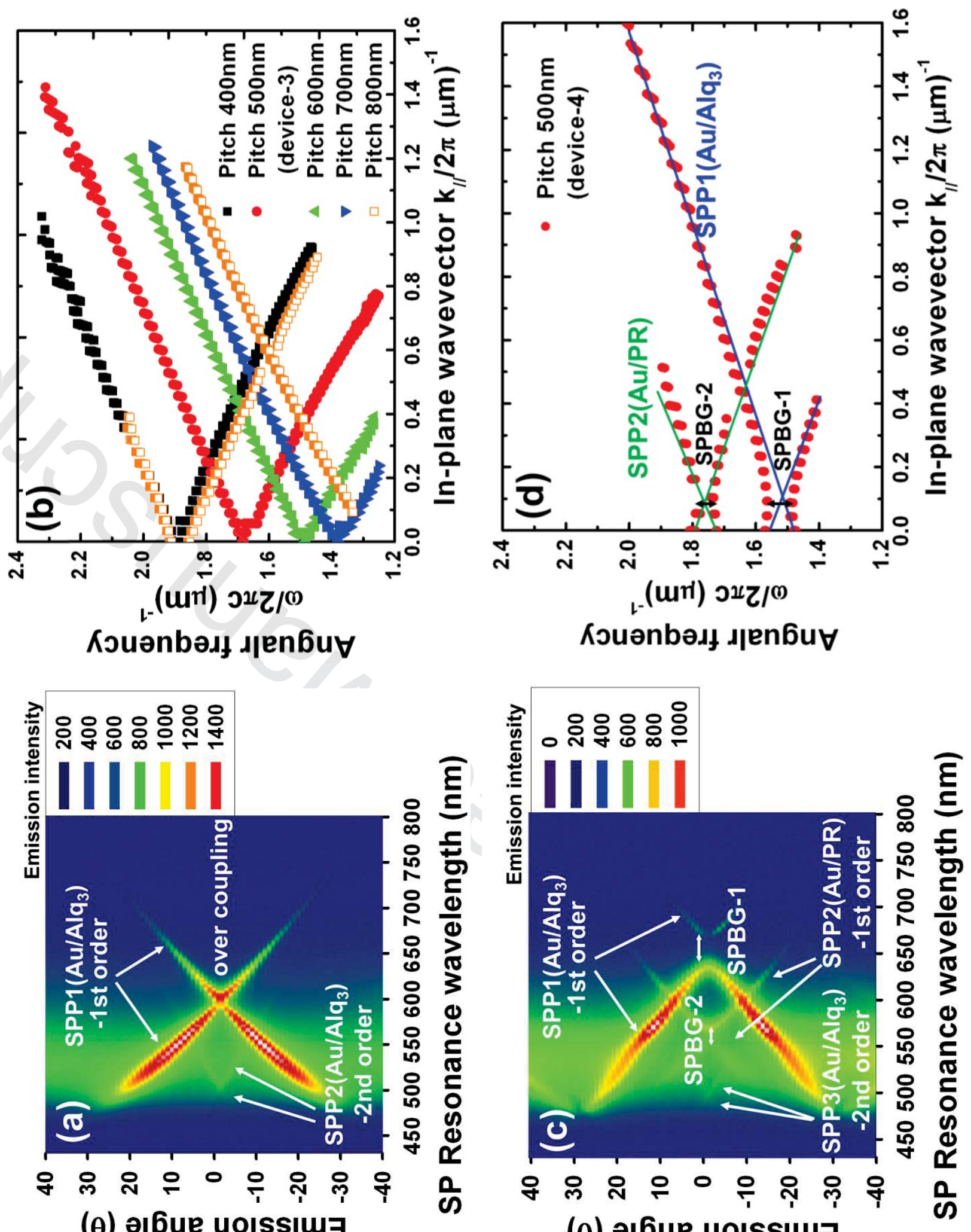

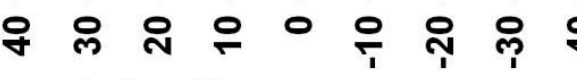

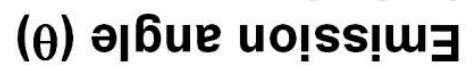

$(\theta)$ әбbue uo!ss!m 